\title{
Epidemiological Profile and Antimicrobial Resistance Pattern of Enteric Fever in a Tertiary Care Hospital of North India - a Seven Year Ambispective Study
}

\author{
Anuradha Makkar1, Shilpi Gupta², Inam Danish Khan',*, Rajiv Mohan Gupta33, KS Rajmohan, \\ Harleen Chopra ${ }^{1}$, Manisha Gupta ${ }^{4}$, Sachin Bansal ${ }^{5}$, Bindu Poonia ${ }^{1}$, Muqtadir Malik³, \\ Pragyan Swagatika Panda ${ }^{1}$
}

\begin{abstract}
Introduction: Enteric-fever is a major public-health problem in developing countries emerging as multidrug-resistant, Nalidixic-acid resistant and extremely drug-resistant Salmonella (Pakistan, 2016), has intensified the use of WHO watch/reserve group antimicrobials such as azithromycin and meropenem.

Methods: This ambispective-study was conducted on 782 non-repeat blood-culture isolates of S. Typhi, S. Paratyphi A and S. Paratyphi B obtained from 29,184 blood cultures received at a 1000-bedded tertiary-care hospital of North-India from 2011-2017. Identification and antibiograms were obtained by Vitek-2 compact and Kirby-Bauer's disc diffusion with resistance to ampicillin, chloramphenicol and cotrimoxazole being labeled as multidrug-resistant. Decreased ciprofloxacin-susceptibility and ciprofloxacin-resistance were defined as MIC $0.125-0.5$ and $>1 \mu \mathrm{g} / \mathrm{ml}$.

Results: S. Typhi and S. Paratyphi A in a ratio of 3.9:1 were seen between July-September predominantly distributed between 6-45 year age group. Resistance to co-trimoxazole, chloramphenicol, ceftriaxone and azithromycin was $6.1 \%, 13.8 \%, 16.1$ and $5.78 \%$ respectively. Multidrug-resistant S. typhi and S. paratyphi $A$ were $2.73 \%$ and $1.91 \%$ respectively.

Conclusion: Enteric-fever is a major public-health problem in India. Emergence of multidrug-resistant, Nalidixic-acid resistant and extremely-drug resistant Salmonella mandates ongoing surveillance for targeted empirical therapy and containment of spread. Repeated epidemics call for water, sanitation, hygiene and vaccination strategies to sustain herd-immunity.
\end{abstract}

\section{KEYWORDS}

antimicrobial resistance; enteric fever; multidrug-resistant Salmonella

AUTHOR AFFILIATIONS

${ }^{1}$ Army College of Medical Sciences and Base Hospital, New Delhi, India

2 Department of Microbiology, MH Bhopal, India

${ }^{3}$ Army Hospital Research and Referral, New Delhi 110010, India

${ }^{4}$ Sanjay Gandhi Post-Graduate Institute, Lucknow, India

${ }^{5}$ Dr Lal Path Labs, Tilak nagar, New Delhi, India

* Corresponding author: Microbiology and Infectious Diseases, Army College of Medical Sciences and Base Hospital, New Delhi 110010, India; e-mail: titan_afmc@yahoo.com

Received: 9 August 2018

Accepted: 14 November 2018

Published online: 22 January 2019

Acta Medica (Hradec Králové) 2018; 61(4): 125-130

https://doi.org/10.14712/18059694.2018.130

(c) 2018 The Authors. This is an open-access article distributed under the terms of the Creative Commons Attribution License (http://creativecommons.org/licenses/by/4.0), which permits unrestricted use, distribution, and reproduction in any medium, provided the original author and source are credited. 


\section{INTRODUCTION}

Enteric-fever is one of the major public health problems in developing countries including India where safe drinking water and sanitation is not warranted (1). According to the estimates, worldwide there are 12-33 million new patients of enteric-fever annually with mortality reaching 600,000 (2). Typhoid is highly endemic in South Asia, South-East Asia, sub-Saharan Africa and West coast of Latin America. The incidence of enteric-fever range from 102 to 2,219 per 100,000 population in India and 9.8 cases per 1000 person-years in Delhi. The estimated annual incidence rate of $1 \%$ in India (3).

Enteric-fever constitutes typhoid fever caused by Salmonella enterica subsp enterica serotype Typhi and paratyphoid fever caused by Salmonella enterica subsp enterica serotypes Paratyphi A, Paratyphi B and Paratyphi C. Several studies from India have reported $S$. Typhi as the most common causative agent, along with increasing number of patients due to S. Paratyphi A. Amongst the changing trends in epidemiology of enteric-fever one of the significant findings is increased incidence of S. Paratyphi A infections. Several studies from India have reported the increased incidence of S. Paratyphi A infections since 1996. However, the incidence of S. Paratyphi $B$ and $C$ are rarely reported from India (4-6). Usually majority of enteric-fever patients occur in children less than 15 years of age, but children younger than five years of age are more susceptible to infection in disease endemic areas. On the other hand, studies from North India have reported the incidence of paratyphoid fever more commonly in adults $(4,7)$. However, as far as seasonal distribution of enteric-fever is concerned there is not much variation within the country. The studies from North India have encountered the occurrence of patients throughout the year with the peak of disease usually observed during summer and rainy season (8).

The case-fatality rate is $10-50 \%$ due to complications such as perforated typhoid ulcer, Myocarditis and shock; with 1-5 year children being at the highest risk. Mortality rates due to enteric-fever can be reduced from $30 \%$ to less than $1 \%$ by providing effective antimicrobial therapy which is facing challenges due to emerging antimicrobial resistance (9). Ampicillin, chloramphenicol and trimethoprim-sulphamethoxazole were the conventional first line drugs for enteric-fever till mid-twentieth century. Since 1962, E. coli integron carrying resistance genes acquired through plasmids has appeared worldwide. After emergence of chloramphenicol resistant S. Typhi in India and Mexico in 1972, frequency of isolation of multidrug resistant (MDR) Salmonella is on increase worldwide $(10,11)$. Since 1989 , there have been several outbreaks of MDR salmonella pathogens reported from Asian countries including India (12). With the increased prevalence of MDR isolates, third generation cephalosporins and fluoroquinolones are recommended for MDR infections. Nalidixic acid resistant ( $\mathrm{NaR}$ ) Salmonella have emerged due to point mutations in gyrA gene leading to ten-folds higher MICs compared to fully susceptible strains. Recent emergence of extremely drug resistant Salmonella resistant to ceftriaxone and ciprofloxacin has intensified the use of WHO watch/reserve group antimicrobials such as azithromycin and meropenem (13). It is important to study the prevalence, epidemiological factors and antibiogram related to enteric-fever to enable effective treatment and preventive measures in terms of vaccines and strengthening hygiene and sanitation measures. This ambispective study was undertaken to study the prevalence, epidemiology and antimicrobial resistance pattern of S. Typhi and S. Paratyphi species in a tertiary-care teaching hospital in North India.

\section{MATERIALS AND METHODS}

An ambispective study was conducted on 645 non-repeat blood-culture isolates of S. Typhi, S. Paratyphi A and S. Paratyphi B isolated at a 1000-bedded tertiary care hospital of North India from January 2011 to December 2017 after inferences from a pilot study conducted for the period covering Jul-Sep 2010 and due approval from the Hospital Ethics Committee. The pilot study was conducted to improve identification of Salmonella using automated systems for blood culture, microbiological identification and susceptibility.

All samples were plated directly on blood and MacConkey agar after positive culture screen from BacT/ALERT ${ }^{\circledR} 3 \mathrm{D}$ (bioMérieux SA, F-69280 Marcy l’Etoile, France) blood culture system and incubated at $37^{\circ} \mathrm{C}$ for $18-24 \mathrm{hrs}$. Identification and antimicrobial susceptibility testing of the isolates were done by Vitek 2 compact (bioMérieux SA, France). Inbuilt standards for identification comparison were utilized. Identification percentage $>85 \%$ and Vitek Advanced Expert System flagging consistent were taken as cut-off for final validation. Isolates were also tested by slide agglutination using specific sera.

In parallel manual antimicrobial susceptibility was put for azithromycin $(15 \mu \mathrm{g})$ and chloramphenicol $(30 \mu \mathrm{g})$ (HiMedia laboratories, India) using Kirby-Bauer's disc diffusion method as these antimicrobials are not covered in susceptibility panel for gram negative bacilli in Vitek 2 compact. All interpretations were done using CLSI 2016 guidelines. In 2012, the CLSI revised the breakpoints for ciprofloxacin susceptibility for typhoidal Salmonella with MIC ranging from $\leq 0.06 \mu \mathrm{g} / \mathrm{ml}$ (susceptible) to $\geq 1 \mu \mathrm{g} / \mathrm{ml}$ (resistant) as compared to earlier MIC range from $\leq 1 \mu \mathrm{g} / \mathrm{ml}$ (susceptible) to $\geq 4 \mu \mathrm{g} / \mathrm{ml}$ (resistant). Interpretation of ciprofloxacin for all the isolates including 2011 was done according to the revised CLSI guidelines of 2012 (14). Decreased ciprofloxacin susceptibility (DCS) was defined as isolates having MIC of ciprofloxacin within range of $0.125-0.5 \mu \mathrm{g} / \mathrm{ml}$. Ciprofloxacin resistant strains were defined for isolates with MIC $>1 \mu \mathrm{g} / \mathrm{ml}$. Isolates resistant to ampicillin, chloramphenicol and co-trimoxazole (trimethoprim-sulphamethoxazole were labeled as MDR.

\section{RESULTS}

A total of $780(2.67 \%)$ isolates of typhoidal Salmonella were isolated from 29,184 blood cultures received during the study period. The number of S. Typhi isolated was 623 (79.67\%) compared to 157 (20.1\%) S. Paratyphi $A$ and two S. Paratyphi $B(0.26 \%)$ isolates (Table 1$)$. Male : female ratio was $2.3: 1$. The highest number of culture positive enteric-fever patients were seen in the age groups of $6-15$ years and $16-45$ years with $345(44.23 \%)$ and $346(44.36 \%)$ isolates respectively. (Table 2) 
Tab. 1 Distribution of S. Typhi, S. Paratyphi A and S. Paratyphi B in blood samples.

\begin{tabular}{|c|c|c|c|c|c|}
\hline & No. of blood samples & S. Typhi & S. Paratyphi A & S. Paratyphi B & Total \\
\hline 2011 & 3010 & $64(2.1)$ & $9(0.29)$ & - & 73 \\
\hline 2012 & 3420 & $79(2.3)$ & $13(0.38)$ & - & 92 \\
\hline 2013 & 3860 & $73(1.9)$ & $11(0.28)$ & - & 84 \\
\hline 2014 & 4468 & $120(2.7)$ & $35(0.78)$ & 1 & 156 \\
\hline 2015 & 4476 & $79(1.76)$ & $18(0.4)$ & - & 97 \\
\hline 2016 & 4850 & $109(2.24)$ & $33(0.68)$ & 1 & 143 \\
\hline 2017 & 5100 & 99 (1.94) & $38(0.74)$ & - & 137 \\
\hline Total & 29184 & $623(2.13)$ & $157(0.53)$ & 2 & 782 \\
\hline
\end{tabular}

Tab. 2 Age wise distribution of S. Typhi, S. Paratyphi A and S. Paratyphi B in blood samples.

\begin{tabular}{|l|l|l|l|l|}
\hline Age (years) & S. Typhi & S. Paratyphi A & S. Paratyphi B & Total \\
\hline$\leq 5$ & 48 & 3 & - & 51 \\
\hline $6-15$ & 279 & 66 & - & 345 \\
\hline $16-45$ & 259 & 85 & 2 & 346 \\
\hline$>46$ & 37 & 3 & - & 40 \\
\hline Total & 623 & 157 & 2 & 782 \\
\hline
\end{tabular}

Tab. 3 Cumulative percentage susceptibility of S. Typhi and S. Paratyphi A towards various antimicrobials.

\begin{tabular}{|l|l|l|}
\hline Antimicrobials & $\begin{array}{l}\text { S. Typhi } \\
(\mathbf{n = 6 2 3 ) ( \% )}\end{array}$ & $\begin{array}{l}\text { S. Paratyphi A } \\
(\mathbf{n}=157)(\%)\end{array}$ \\
\hline Ampicillin & $489(78.49)$ & $106(67.52)$ \\
\hline Ceftriaxone & $599(96.14)$ & $130(82.8)$ \\
\hline Nalidixic acid & $320(51.36)$ & $81(51.59)$ \\
\hline Ciprofloxacin & $176(28.25)$ & $7(4.45)$ \\
\hline Co-trimoxazole & $585(93.9)$ & $146(92.99)$ \\
\hline Azithromycin & $587(94.22)$ & $144(91.71)$ \\
\hline Chloramphenicol & $537(86.19)$ & $117(74.52)$ \\
\hline
\end{tabular}

Tab. 4 Mapping of resistance phenotypes of S. Typhi and S. Paratyphi A.

\begin{tabular}{|c|c|c|c|c|c|}
\hline & AMR groups & $\begin{array}{l}\text { MDR } \\
\text { n (\%) }\end{array}$ & $\begin{array}{l}\text { NAR } \\
\text { n (\%) }\end{array}$ & $\begin{array}{l}\text { DCS } \\
\text { n (\%) }\end{array}$ & CiR \\
\hline \multirow[t]{2}{*}{2011} & S. Typhi (64) & $2(3.12 \%)$ & $4(6.25 \%)$ & $37(57.81 \%)$ & $24(37.5 \%)$ \\
\hline & S. Paratyphi A (9) & 0 & $5(55.55 \%)$ & 0 & $9(100 \%)$ \\
\hline \multirow[t]{2}{*}{2012} & S. Typhi (79) & $3(3.79)$ & $20(25.31 \%)$ & $50(63.29)$ & $27(34.17)$ \\
\hline & S. Paratyphi A (13) & $1(7.69 \%)$ & $1(7.69 \%)$ & 0 & $12(92.3)$ \\
\hline \multirow[t]{2}{*}{2013} & S. Typhi (73) & $4(5.47 \%)$ & $28(38.35 \%)$ & $39(53.42)$ & $31(42.46)$ \\
\hline & S. Paratyphi A (11) & $1(9.09 \%)$ & $3(27.27 \%)$ & 0 & $11(100)$ \\
\hline \multirow[t]{2}{*}{2014} & S. Typhi (120) & $3(2.5 \%)$ & $67(55.83 \%)$ & $69(57.5)$ & $49(40.83)$ \\
\hline & S. Paratyphi A (35) & 0 & $7(20 \%)$ & $1(2.85)$ & $32(91.42)$ \\
\hline \multirow[t]{2}{*}{2015} & S. Typhi (79) & 0 & $32(40.5 \%)$ & $49(62.02)$ & $22(27.84)$ \\
\hline & S. Paratyphi A (18) & 0 & $9(50 \%)$ & 0 & $16(88.88)$ \\
\hline \multirow[t]{2}{*}{2016} & S. Typhi (109) & $2(1.83 \%)$ & $104(95.41 \%)$ & $62(56.88)$ & $41(37.61)$ \\
\hline & S. Paratyphi A (33) & 0 & $33(100 \%)$ & 0 & $27(81.81)$ \\
\hline \multirow[t]{2}{*}{2017} & S. Typhi (99) & $2(2.02 \%)$ & $89(89.9 \%)$ & $53(53.53)$ & $36(36.36)$ \\
\hline & S. Paratyphi A (38) & 0 & $30(90.9 \%)$ & 0 & $29(76.31)$ \\
\hline
\end{tabular}


Seasonal occurrence was determined by dividing the year into four quarters. Although enteric-fever occurred in all months throughout the year, the cumulative maximum number of S. Typhi occurred during July-September (47.51\%) followed by April-June (33.1\%). The peak incidence of S. Paratyphi was also observed in July-September (45.86\%) followed by April-June (21.66\%) (Fig. 1, 2).
Resistance to co-trimoxazole, chloramphenicol, ceftriaxone and azithromycin was $6.1 \%, 13.8 \%, 16.1$ and $5.78 \%$ respectively. MDR S. typhi and S. paratyphi A were $2.73 \%$ and $1.91 \%$ respectively (Table 3 ). Table 4 shows the year wise distribution of four major resistance phenotypes analysed for Salmonella: MDR, NaR, DCS and ciprofloxacin resistant (CiR) S. Typhi and S. Paratyphi A.

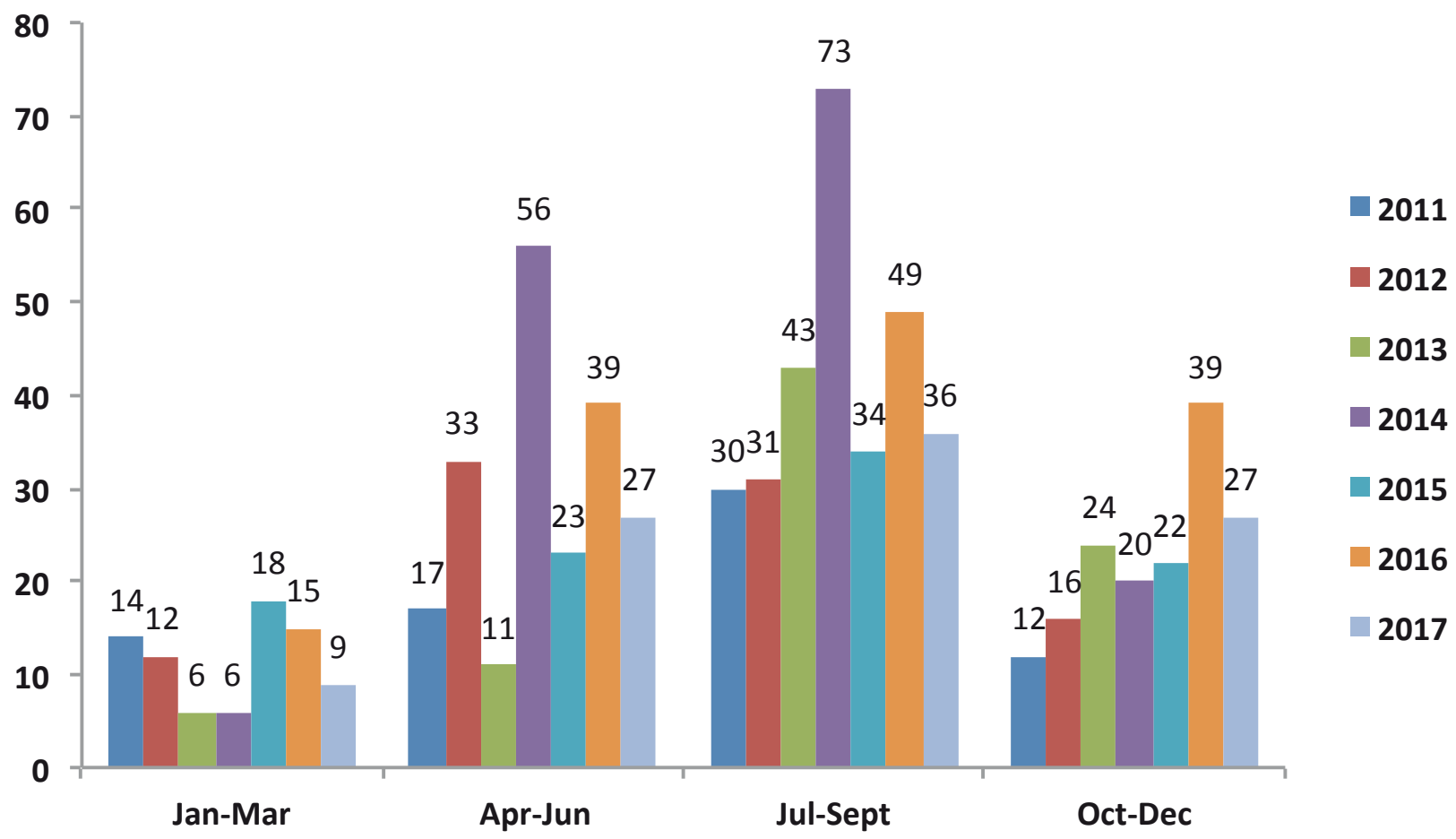

Fig. 1 Epidemiological mapping of S. Typhi at a 1000-bedded tertiary-care teaching hospital in New Delhi from 2011-2017.

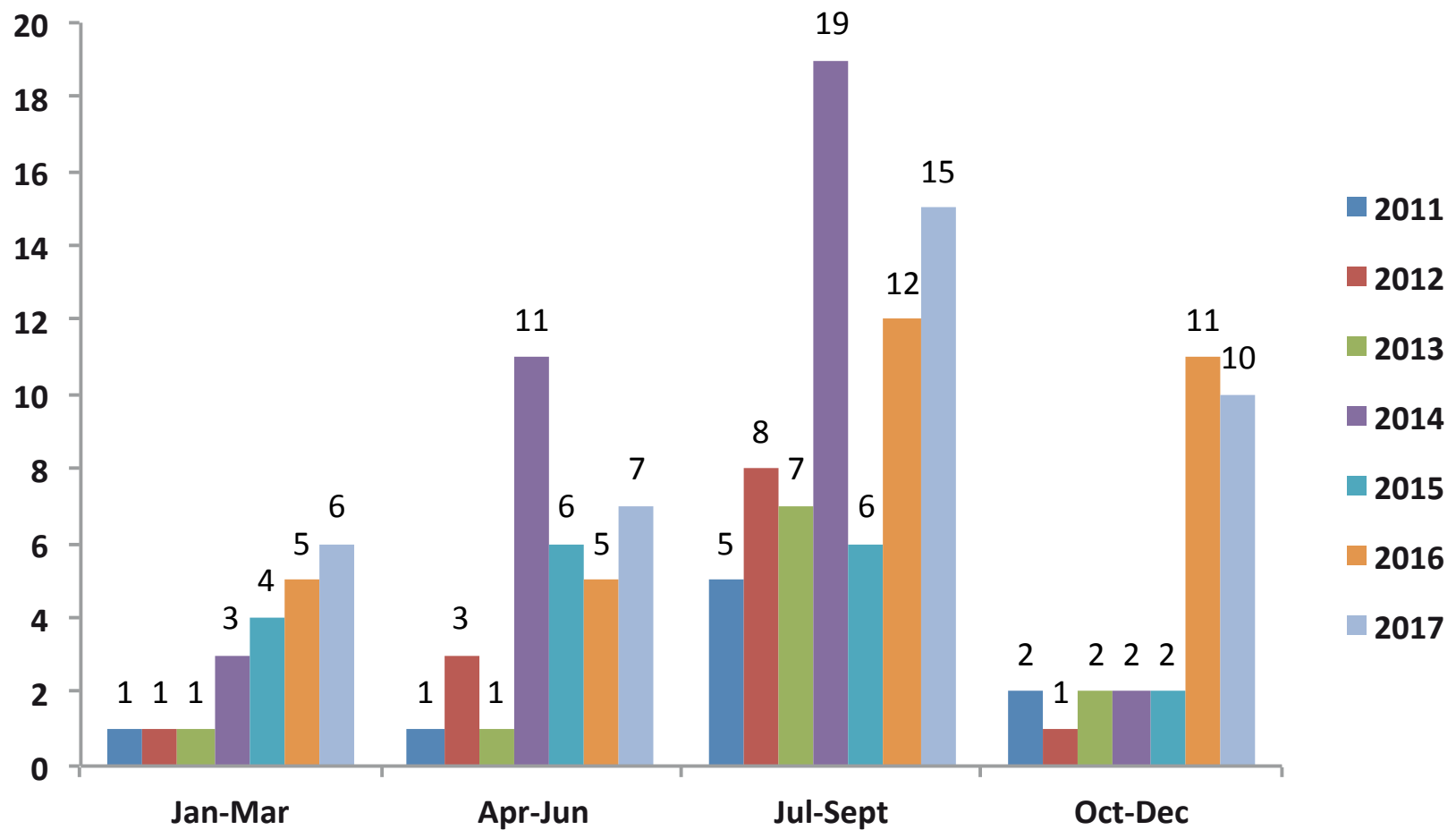

Fig. 2 Epidemiological mapping of S. Paratyphi A at a 1000-bedded tertiary-care teaching hospital in New Delhi from 2011-2017. 


\section{DISCUSSION}

Enteric-fever continues to be a major public health problem in India and the present study was undertaken to estimate the magnitude of this problem in western part of the country. In the present prospective study conducted from 2011-2017, S. Typhi and S. Paratyphi A were isolated for a total of $81.2 \%$ and $18.4 \%$ of enteric-fever patients respectively. Thus the ratio of isolation of S. Typhi and S. Paratyphi A was $4.4: 1$ which was in accordance to several studies conducted in various parts of the country who reported ratio from $1.6: 1$ to $4: 1$ (8, 15-17). Though the incidence of S. Typhi remains high, several recent studies have highlighted the progressive increased proportion of S. Paratyphi A in the past decade $(18,19)$. However, this is in contrast to our finding where incidence of S. Paratyphi A remained almost constant throughout the duration of the study with random increase during the year 2014 and 2016.

The peak incidence of enteric-fever is seen in school aged children between 5-15 years of age group and in young adults. After this age the incidence falls probably due to acquired immunity resulting from clinical or subclinical infection (8). Our finding in the current study is in collaboration with this fact where we found maximum occurrence of enteric-fever patients in the age group 6-15 years. However, $58.8 \%$ of the isolated S. Paratyphi A was found in $16-45$ years of the age group which was in accordance with other studies who have reported paratyphoid fever more commonly in adults (4, 7). Several studies from Indian subcontinent have reported peaks of transmission of Salmonella serotypes either in dry season (April-June) or in rainy weather (July-Sept). Both warm and rainy season favour the growth of the micro-organisms. During dry weather, due to the lowering of water level stagnation of water deteriorates the quality resulting in increased chances of contamination $(4,7,8)$. We report similar findings where the maximum number occurred during July-September followed by April-June both in patient of S. Typhi and S. Paratyphi A.

Increase in the incidence of MDR Salmonella resistant to ampicillin, chloramphenicol, cotrimoxazole, streptomycin, furazolidone and tetracyclines is an emerging problem and a matter of concern worldwide $(8,17)$. The emergence of antimicrobial resistance in Salmonella holds same risk factors as for any other bacteria. MDR is acquired from animals through food chain. In addition, uncontrolled use of antimicrobials in veterinary medicine is also a major contributor in emergence of resistance in Salmonella (20). In the last decade there are several studies from various regions of India who have reported high rate of MDR typhoid fever ranging from 6.84-66.6\% (21-23). Outbreaks of MDR typhoid have occurred in Kerala, Mumbai and Delhi frequently affecting the pediatric age group. However in the present decade reversal in the resistance trends in Salmonella is reported by many Indian centres $(24,25,26)$. A recent surveillance study conducted by ICMR across four tertiary care centres in India have also reported MDR rate less than $3 \%(27)$. In the present study also, there appears to be high susceptibility rate towards first line antibiotics, thus accounting for low rate of MDR strains. Though with reduced MDR rates and increased susceptibility with first line drugs, it is tempting to recycle old drugs but due to the several reasons it is unlikely in India in near future. Loss of confidence in first line antibiotics amongst clinicians due to occurrence of more cases of recurrence and relapse and severe complications like aplastic anaemia associated with these chloramphenicol marks the top most reasons (26).

According to the National Treatment Guidelines for antimicrobial use in infectious diseases published by National Centre for Disease Control (NCDC) in 2016, Azithromycin is recommended for uncomplicated disease and ceftriaxone for patients who require parenteral therapy. The present study reports $93.6 \%$ susceptibility of Azithromycin. Azithromycin is indicated for MDR, NaR and empirical therapy as it attains high concentrations for intracellular Salmonella. It is also helpful in treating many other etiologies of undifferentiated fever like leptospirosis, scrub typhus, lower respiratory tract infections (LRTI) which has a definite advantage in areas where there is lack of accurate diagnosis (26). Studies conducted on susceptibility pattern of Azithromycin against Salmonella serotypes in Delhi and Lucknow found susceptibility rate to be $92 \%$ and $92.6 \%$ respectively $(28,29)$. However Dutta et al reported $28 \%$ of S. Paratyphi A resistant to Azithromycin (17).

With the emergence of MDR strains, rampant use of Ciprofloxacin resulted in DCS and CiR strains (27). Resistance and/or reduced susceptibility in flouroquinolones is associated with point mutation in topoisomerase or gyrase gene, which gives rise to DCS. DCS strains are associated with delayed clinical response and increase in complications. Accumulation of further mutations with or without added efflux pumps, lead to CiR (26). In this study, we found $58.4 \%$ and $0.84 \%$ of DCS and $37 \%$ and $89.9 \%$ of CiR strains among S. typhi and S. paratyphi respectively. Similar to our findings, several studies from India have reported high rate of resistance to Ciprofloxacin $(23,26,28)$.

With the present reports of clinical failure with ciprofloxacin, third generation cephalosporins such as ceftriaxone have become drug of choice. Recently, there have been sporadic reports of ceftriaxone resistance in Salmonella which is known to be associated with acquisition of Extended Spectrum $\beta$-lactamases (ESBL) genes. India, Pakistan, Bangladesh, Iran, Iraq, Egypt and Philippines are endemic for ESBL Salmonella thereby threatening prognosis of typhoid among residents as well as travelers. Salmonela also exhibit CTX-M, CMY, OXA, SHV, and TEM $\beta$-lactamases. The percentage of ceftriaxone resistant S. Typhi (4.1\%) and S. Paratyphi (17.6\%) strains in the present study is a matter of concern. In contrast several Indian studies have reported $100 \%$ susceptibility of Salmonella isolates towards ceftriaxone $(23,27,28)$.

However, Bhattacharya et al have reported $6.25 \%$ ceftriaxone resistance in Western Orissa (15). In present study the MIC values of 10 (1.9\%) and 11 (9.2\%) strains of S. Typhi and S. Paratyphi A were found in the range between 8 to $64 \mu \mathrm{g} / \mathrm{ml}$. An increase in ceftriaxone MIC has been also observed by $\mathrm{Da}$ hiya et al. (29).

A recent study from Sindh, Pakistan has highlighted the emerging issue of 300 extensively drug resistant (XDR) Salmonella amongst 850 infections and four deaths since November 2016 (30). Global spread of this XDR strain susceptible only to Azithromycin is contemplated to threaten developed world countries due to international travel. However, there are limited studies on susceptibility patterns of azithromycin as it is not being used routinely. 
Infection prevention and control of Salmonella requires interventions at reservoir, transmission and host fronts. Reservoir elimination should be focused on provisioning of clean potable water, hygienic food. Chain of transmission can be broken by emphasis of hand-hygiene amongst chefs, food handlers and kitchen staff. Outbreak monitoring systems should be instituted to implement swift anti-epidemic measures. Continued emergence of multidrug-resistant, $\mathrm{Na}$ lidixic-acid resistant and extremely drug-resistant Salmonella mandates ongoing surveillance to type antibiogram patterns for empirical therapy and containment of spread.

\section{CONCLUSION}

Enteric-fever continues to be a major public-health problem in India. Emergence of multidrug-resistant and extremely drug-resistant Salmonella mandates ongoing surveillance to type antibiogram patterns for empirical therapy and containment of spread. Repeated epidemics call for implementation of preventive measures like water, sanitation and hygiene (WASH) along with pre-emptive vaccination strategies to sustain herd immunity in susceptible communities.

\section{REFERENCES}

1. Crump JA, Luby SP, Mintz ED. The global burden of typhoid fever. Bull World Health Org 2004; 82: 346-53.

2. Miller SI, Pegues DA. Salmonella species, including Salmonella typhi. In: Mandell GL, Bennet JE, Dolin R, eds. Principles and Practice of Infectious Disease, 4th edn. New York: Churchill Livingstone, 1998: pp. 2344-73.

3. Chowta MN, Chowta NK. Study of clinical profile and antibiotic response in typhoid fever. Indian J Med Microbiol 2005; 23: 125-27.

4. Kanung OS, Dutta S, Sur D. Epidemiology of typhoid and paratyphoid fever in India. J Infect Developing Countries 2008; 2(6): 454-60.

5. Tankhiwale SS, Agrawal G, Jalgaonkar SV. An unusually high occurrence of Salmonella enterica serotype Paratyphi A in patients with enteric fever. Indian Journal of Medical Research 2003; 117: 10-2.

6. Mahapatra A, Patro S, Choudhury S, Padhee A, Das R. Emerging enteric fever due to switching biotype of Salmonella (Paratyphi A) in Eastern Odisha. Indian J Pathol Microbiol 2016; 59: 327-9.

7. Sur D, Ali M, von Seidlein L, et al. Comparisons of predictors for typhoid and paratyphoid fever in Kolkata, India. BMC Public Health 2007; 7(147): 289.

8. Mohanty S, Renuka K, Sood S, Das BK, Kapil A. Antibiogram pattern and seasonality of Salmonella serotypes in a North Indian tertiary-care hospital. Epidemiol. Infect 2006; 134: 961-6.

9. Maskey AP, Basnyat B, Thwaltes GE, et al. Emerging trends in enteric fever in Nepal: 9124 cases confirmed by blood culture 1993-2003. Trans R Soc Trop Med Hyg 2008; 102: 91-5.

10. Panikar CKJ, Vimala KN. Transferable chloramphenicol resistance in Salmonella typhi. Nature 1972; 239: 109-10.

11. Olarte J, Galind DE. Salmonella typhi resistant to chloramphenicol, ampicillin and other antimicrobial agents: Strains isolated during an extensive typhoid fever epidemic in Mexico. Antimicrob Agents Chemother 1973; 4: 597-601.
12. Chuang $\mathrm{CH}$, Su LH, Perera J, et al. Surveillance of antimicrobial resistance of Salmonella enterica serotype typhi in seven Asian countries. Epidemiology and Infection 2009; 137: 266-9.

13. Sin L, Cariappa MP. Blood culture isolates and Antibiogram of Samonella: Experience of a tertiary care hospital. Med J Armed Forces India 2016; 72 (1): 281-4.

14. Clinical and Laboratory Standards Institute. Performance Standards for Antimicrobial Susceptibility Testing: Twenty Second Informational Supplement M100-22. Wayne, Pennsylvania: Clinical and Laboratory Standards Institute; 2012.

15. Bhattacharya SS, Das U, Choudhury BK. Occurrence \& Antibiogram Salmonella Typhi \& S. Paratyphi A isolated from Rourkela, Orissa. Ind J Med Microbiol 2011; 133: 431-3.

16. World Health Organization: Indian Network for Surveillance of Antimicrobial Resistance. Antibiogram of Salmonella enterica serovar Typhi and Salmonella enterica serovar Paratyphi A: a multi-centre study from India. WHO South-East Asia J Public Health 2012; 1: 182-8.

17. Dutta S, Das S, Mitra U, et al. Antimicrobial Resistance, Virulence Profiles and Molecular Subtypes of Salmonella enterica Serovars Typhi and Paratyphi A Blood Isolates from Kolkata, India during 2009-2013. PLoS ONE 2014; 9(8): e101347.

18. Gupta V, Kaur J, Chander J. An increase in enteric fever cases due to Salmonella paratyphi A in and around Chandigarh. Indian J Med Res 2009; 129: 95-8.

19. Bhattacharya SS, Dash U. A sudden rise in occurrence of Salmonella paratyphi A infection in Rourkela Orissa. Indian J Med Microbiol 2007; 25: 78-9.

20. Zaki SA, Karande S. Multidrug resistant typhoid fever: a review. J Infect Dev Ctries 2011; 5(5): 324-37.

21. Shu-Kee E, Pusparajah P, Ab Mutalib NS, et al. Salmonella: A review on pathogenesis, epidemiology and antibiotic resistance. Frontiers in Life Science 2015; 8(3): 284-93.

22. Jog S, Soman R, Singhal T, et al. Enteric fever in Mumbai-clinical profile, sensitivity patterns and response to antimicrobials. J Assoc Physicians India 2008; 56: 237-40.

23. Sen B, Dutta S, Sur D, et al. Phage typing, biotyping \& antimicrobial resistance profile of Salmonella enterica serotype Typhi from Kolkata. Indian J Med Res 2007; 125: 685-8.

24. Kumar Y, Sharma A, Mani KR. Antibiogram Profile of Salmonella enterica Serovar Typhi in India - a two year study. Trop Life Sci Res 2013: 24: 45-54.

25. Badiyal A, Kumar Y, Sharma A, Mani KR. Re-emergence of chloramphenicol sensitive isolates of Salmonella enterica serovar typhi isolates in India during 2013-14. Int J Current Microbiol Appl Sci 2015: 4: 1081-6.

26. Divyashree $S$, Nabarro LEB, Veeraraghavan B and Rupali P. Enteric fever in India: current scenario and future directions. Tropical Medicine and International Health 2016; 21: 1255-62.

27. Dahiya S, Sharma P, Kumari B, et al. Characterisation of antimicrobial resistance in Salmonellae during 2014-2015 from four centres across India: An ICMR antimicrobial resistance surveillance network report. Indian J Med Microbiol 2017; 35: 61-8.

28. Saksena R, Nayyar C, Manchanda V. Six-year susceptibility trends and effect of revised Clinical Laboratory Standards Institute breakpoints on ciprofloxacin susceptibility reporting in typhoidal Salmonellae in a tertiary care paediatric hospital in Northern India. Indian J Med Microbiol 2016; 34: 520-5.

29. Misra R, Prasad KN. Antimicrobial susceptibility to Azithromycin among Salmonella enteric typhi and Paratyphi A isolates from India. J Med Microbiol 2016; 65(12): 1536-9.

30. Klemm EJ, Shakoor S, Page AJ, et al. Emergence of an extensively drug resistant Salmonella enteric serovar Typhi clone harbouring a promiscuous plasmid encoding resistance to flouroquinolones and third generation cephalosporins. mBio 2018; 9: e00105-18. 